\title{
Philosophiques
}

\section{Penser les relations internationales d'un point de vue féministe}

\section{RYOA CHUNG}

Volume 44, numéro 1, printemps 2017

URI : https://id.erudit.org/iderudit/1040334ar

DOI : https://doi.org/10.7202/1040334ar

Aller au sommaire du numéro

Éditeur(s)

Société de philosophie du Québec

ISSN

0316-2923 (imprimé)

1492-1391 (numérique)

Découvrir la revue

Citer ce document

Chung, R. (2017). Penser les relations internationales d'un point de vue

féministe. Philosophiques, 44(1), 131-138. https://doi.org/10.7202/1040334ar d'utilisation que vous pouvez consulter en ligne.

https://apropos.erudit.org/fr/usagers/politique-dutilisation/ 


\title{
Penser les relations internationales d'un point de vue féministe
}

\author{
RYOA CHUNG \\ Université de Montréal \\ ryoa.chung@umontreal.ca
}

Le brillant ouvrage de Soumaya Mestiri, Décoloniser le féminisme. Une approche transculturelle (Paris, Vrin, 20I6), ne s'inscrit pas comme tel dans le champ des théories des relations internationales en science politique, ni dans le domaine des théories de justice globale en philosophie politique contemporaine. Mais dans la mesure où les travaux de Mestiri portent sur la nécessité de décoloniser les discours féministes dominants qui prétendent à une portée universalisable et dans la mesure où l'auteure développe une approche qui se veut «transculturelle » en travaillant le concept de frontière, il va de soi que les lecteurs intéressés à la fois par les questions philosophiques inhérentes au féminisme $e t$ aux enjeux éthiques en relations internationales y trouveront matière à réflexion.

Dans ce monde affligé par la violence des rapports de pouvoir entre les États, les populations, les groupes sociaux et les individus, il est impératif de penser la condition des femmes afin d'avoir une compréhension plus exhaustive des modalités contemporaines des inégalités historiques qui ont configuré l'ordre international. Il s'agit d'une affirmation qui repose à la fois sur des observations empiriques ainsi que sur des raisons méthodologiques et épistémologiques.

D'un point de vue empirique, l'étude des conditions d'existence des femmes à travers le monde donne des indications plus précises au sujet des inégalités socioéconomiques à l'échelle internationale. Suivant les travaux de l'économiste A. Sen, pour ne citer que cet exemple mieux connu en philosophie politique, elle permet de mieux comprendre, au-delà de la comparaison du PNB entre les pays, la redistribution des ressources au sein d'une population du point de vue des individus les plus démunis qui sont généralement des femmes et leurs enfants à charge. L'étude des conditions d'existence des femmes permet également de capter des indices plus raffinés de discriminations sociales en pénétrant plus facilement dans la sphère domestique, dans l'économie informelle et dans le monde plus obscur des formes de violences genrées et racisées qui se déploient en arrière-fond de la séparation entre sphère publique et sphère privée.

D'un point de vue épistémologique, les perspectives féministes de recherche impliquent des points de vue sur le monde qui enrichissent notre savoir (pour emprunter la notion à la riche littérature de la standpoint theory). Par "perspectives féministes ", j'entends de manière très générale des angles d'analyse qui s'intéressent aux problématiques sociales genrées et 
qui les identifient explicitement comme constituant des injustices lorsqu'il s'agit de discriminations moralement arbitraires. Ces perspectives féministes permettent d'identifier des formes d'injustices et d'oppression épistémiques (pour emprunter le terme à $\mathrm{K}$. Dotson ${ }^{1}$ ) qui déterminent les angles morts des paradigmes dominants. Force est de constater que les dimensions genrées et racialisées des relations internationales demeurent généralement sous-conceptualisées dans les approches dominantes en science politique et en philosophie politique occidentales. Une des contributions les plus importantes des travaux de Mestiri consiste certainement à présenter des dissensions épistémiques qui donnent à penser.

En dépit de l'intérêt remarquable que représentent les chapitres II, III et IV qui portent plus spécifiquement sur les représentations problématiques de la femme arabe, sur les enjeux liés au féminisme musulman occidental et sur l'examen fascinant de la révolution tunisienne d'un point de vue féministe, l'expertise nous manque pour pouvoir offrir des remarques critiques approfondies. C'est donc sous l'angle plus particulier de l'éthique des relations internationales, qui constitue davantage notre champ de recherches, que ce commentaire critique mettra l'accent sur certains chapitres en particulier, soit les chapitres I, V et VI. Au risque de reconduire une vision partielle des thèses de l'auteure, une certaine trame argumentative permet de relier les trois chapitres identifiés de manière fluide. Notre propos consistera à indiquer les perspectives féministes de recherche que le livre de Mestiri permet de développer dans le domaine plus particulier de l'éthique des relations internationales tout en identifiant les raisons pour lesquelles la proposition de l'auteure, culminant vers une réhabilitation de la notion de care pour secourir les discours féministes de l'empowerment, nous semble problématique.

Dans le cadre du premier chapitre "Les femmes du Kazanistan ", l'auteure offre une lecture critique percutante de la conception rawlsienne du droit des peuples ${ }^{2}$. Le dernier opus de Rawls est problématique à de nombreux égards, mais avant d'aborder les aspects particuliers que Mestiri critique avec raison, il y a lieu de restituer les intentions philosophiques de Rawls de la manière la plus charitable possible (ne serait-ce que par vertu épistémique). Publié en I999, le dernier livre de Rawls fut écrit avant les attentats du World Trade Center qui allaient changer le monde et marquer le commencement d'une ère de "guerre contre le terrorisme" avec le déferlement de l'islamophobie au sein des pays non musulmans qu'on peut y associer. Ce qui ne veut pas dire que l'islamophobie n'existait pas avant cela, au contraire, elle s'est nourrie de l'orientalisme et du colonialisme qui ont perduré pendant des siècles. Mais il s'agit simplement de souligner que Rawls

1. K. Dotson, «Conceptualizing Epistemic Oppression », Social Epistemology, vol. 28, $\mathrm{n}^{\circ} 2,20 \mathrm{I} 4$.

2. Nous suivons simplement ici l'expression que préfère Soumaya Mestiri pour traduire «law of peoples» par droit des peuples plutôt que droit des gens. 
écrivait dans un contexte international tout autre, c'est-à-dire dans le sillage de la Guerre du Golfe (I990-I99I) qui doit elle-même être comprise dans le contexte plus large et complexe du démantèlement du bloc soviétique.

Résistant à un certain triomphalisme libéral qui s'appropriait la fin de la guerre froide et le commencement d'un "nouvel ordre mondial", la conception rawlsienne du droit des peuples peut être interprétée comme une tentative de limiter les ardeurs de l'interventionnisme militaire aux velléités de changement de régime pour des raisons soi-disant humanitaires dans le but de donner un coup de pouce à la démocratisation des États non libéraux. Après tout, comme le cite Rawls lui-même, la "loi de Doyle» ne témoigne-t-elle pas d'une constance empirique démontrant à travers l'histoire moderne des relations internationales que si les États libéraux font la guerre à des États non libéraux, les démocraties libérales ne se font pas la guerre entre elles? Cependant, à l'aune du libéralisme politique caractérisant ce que d'aucuns ont appelé le soi-disant "tournant» de Rawls à partir de la publication de Political Liberalism en 1993 (date à laquelle Rawls présente également la première version de son Law of Peoples dans le cadre des Oxford Amnesty Lectures), c'est le concept de tolérance qui constitue désormais la vertu cardinale du libéralisme que Rawls veut promouvoir à l'échelle internationale, au détriment d'une conception plus substantielle des libertés fondamentales découlant de l'individualisme normatif et de ses implications égalitaires qui avait caractérisé sa théorie de la justice domestique. Face au fait du pluralisme exacerbé en relations internationales, le consensus par recoupement doit éviter les controverses métaphysiques insolubles au sujet des doctrines particulières du bien qui caractérisent l'identité culturelle des peuples, considérés ici comme des acteurs politiques avec lesquels les principes fondamentaux du droit des gens doivent être contractés. C'est dans ce cadre théorique que Rawls présente sa typologie heuristique des sociétés minimalement décentes qui comprennent à la fois des démocraties libérales et des sociétés hiérarchiques bien ordonnées, lesquelles, sans être libérales pour autant, ont droit au respect le plus fondamental des prérogatives légitimes de leur souveraineté. Dans la version finale de I999, l'ouvrage comprend une deuxième partie consacrée au second temps de la théorie non idéale dans laquelle figure donc le Kazanistan inventé par Rawls:

Un des objectifs de la deuxième partie est de montrer qu'il peut exister des peuples non libéraux décents qui acceptent et respectent le Droit des Peuples. Dans cette perspective, j'imagine l'exemple d'un peuple musulman non libéral que j'appelle le "Kazanistan". Ce peuple remplit les critères des peuples hiérarchiques décents: le Kazanistan n'est pas agressif envers les autres peuples, il accepte et applique le Droit des Peuples; il reconnaît et respecte les droits de l'homme; sa structure de base contient une hiérarchie consultative décente, dont je décris les caractéristiques ${ }^{3}$.

3. Rawls, Paix et démocratie. Le droit des peuples et la raison publique, trad. fr. de B. Guillarme, Montréal, Boréal, 2006, p. I7. 
Le propos philosophique de la conception rawlsienne du droit des peuples consiste donc à tenter de reconnaître le pluralisme en relations internationales et à appliquer les principes du libéralisme politique en l'absence d'une structure de base à l'échelle globale (qui limite également l'extrapolation internationale des principes de justice distributive). En dépit de cette idée extrêmement importante qui caractérise sans doute la contribution originale de Rawls dans le domaine de l'éthique internationale, l'essai Paix et démocratie... demeure problématique à de nombreux égards. Une critique féministe libérale remettra en cause les raisons pour lesquelles Rawls exclut l'individualisme normatif et ses implications égalitaires à titre de croyances métaphysiques à l'extérieur du consensus par recoupement pour établir les principes de justice politique entre les peuples. Après tout, sous quels aspects les conceptions holistes de la société seraient-elles moins déterminées par des doctrines particulières du bien et des croyances métaphysiques historiquement et culturellement situées que ne l'est l'héritage des Lumières? Le rejet de l'individualisme normatif entraîne des conséquences lourdes pour ce qui est du noyau des droits humains fondamentaux au cœur du consensus par recoupement qui constitue le critère de démarquage des sociétés minimalement décentes. En effet, la conception rawlsienne de la société hiérarchique bien ordonnée n'implique pas la reconnaissance de l'égalité des chances pour l'accès à des positions d'autorité, de sorte qu'une critique féministe libérale aura de bonnes raisons pour mettre en doute la signification politique réelle des processus de consultation qui se limitent à l'expression parfois purement symbolique de la dissension. Toutefois, la critique de Mestiri est plus complexe que cela. En fait, le débat autour des droits des femmes sert également à renforcer les préjugés occidentaux à l'encontre des sociétés non libérales, de sorte que sous le vernis du principe de tolérance en contexte de pluralisme, la conception rawlsienne de la société hiérarchique décente reconduit une vision profondément paternaliste des sociétés musulmanes.

Si le Kazanistan a servi à quelque chose, c'est à entretenir (encore plus) certains préjugés tenaces. Car, à y regarder de plus près, l'on s'aperçoit que la seule information supplémentaire livrée dans la section dévolue à cette société hiérarchique décente est la qualification comme musulmane. De fait, en donnant une certaine réalité à ce type de société, Rawls réussit la gageure d'exacerber l'intolérance à l'égard des sociétés décentes via, précisément, la question du statut des femmes (Mestiri, 2016, p. 32-33).

La démonstration de Mestiri est certes convaincante et la conception rawlsienne du droit des peuples est problématique à tant d'égards qu'il ne reste pas beaucoup d'arsenal théorique pour la défendre. Cependant, un malaise surgit au détour de certaines affirmations en ce qui a trait au multiculturalisme libéral et à l'approche de l'empowerment que l'auteure critique de manière aussi incisive. Si c'est moins le cas pour la critique contre Rawls, il n'en demeure pas moins que certaines critiques de Mestiri semblent mélanger deux catégories d'arguments: les uns étant d'ordre empirique ou sociolo- 
gique, et les autres étant d'ordre conceptuel. En effet, on a parfois l'impression que le travail de déconstruction, certes crucial pour l'avancement des idées, dénonce des détournements idéologiques de sens qui sont plutôt imputables à l'évolution sociologique de la réception et de la dénaturation politique de certaines idées plutôt qu'à une nécessité conceptuelle. Par exemple, c'est une chose de dire, comme l'auteure l'affirme à plusieurs reprises, que le multiculturalisme libéral reconduit une vision non contestée des rapports de pouvoir entre les communautés majoritaires et les communautés minoritaires d'une société. Il ne fait aucun doute que ce travail de déconstruction en vue de "décoloniser» les paradigmes dominants est absolument essentiel, et il se peut fort bien que dans les faits le modèle multiculturaliste ait donné lieu à ce genre de statu quo profondément problématique d'un point de vue politique. Mais la critique repose-t-elle sur un constat empirique (sociologique) ou sur une nécessité conceptuelle?

Pour qu'il s'agisse de la dernière, il faudrait alors démontrer en quoi consistent les vices conceptuels du modèle libéral du multiculturalisme de Kymlicka ou de Taylor, par exemple, pour affirmer que par dessein ou presque, l'argumentation philosophique au fondement du multiculturalisme libéral à la canadienne (pour ne parler que de ce type-là) reconduit nécessairement une forme de domination qui s'ignore. Après tout, le multiculturalisme de Kymlicka consistait à justifier la revendication des droits collectifs à partir d'une conception des libertés individuelles fondamentales gratifiant le droit à une appartenance culturelle en tentant d'éviter les pièges d'une certaine conception du communautarisme qui subordonne conceptuellement les droits individuels, ou d'une certaine conception du républicanisme qui promeut conceptuellement l'effacement des différences visibles au nom d'une certaine interprétation de l'impartialité universalisable. Qui plus est, à l'encontre d'un certain féminisme libéral qui condamnait le multiculturalisme au nom d'une doctrine compréhensive du libéralisme ${ }^{4}$, les Kymlicka et Nussbaum ont malgré tout tenté de défendre une conception du libéralisme politique tentant de protéger les droits collectifs des minorités contre la tyrannie d'une conception particulière du bien promue par celle de la majorité. S'il est fort possible que, dans les faits, les politiques publiques ayant prétendu appliquer le modèle libéral du multiculturalisme ne sont pas allées aussi loin qu'il le faut, en raison de facteurs sociaux et politiques, pour identifier les injustices passées, présentes et futures du colonialisme et du racisme, cela est une chose dont il faut impérativement discuter. Mais c'en est une autre que d'affirmer que le multiculturalisme libéral est philosophiquement corrompu. Si l'hypothèse paraît néanmoins plausible et véritablement stimulante, la démonstration conceptuelle reste à expliciter.

4. L'argument le plus systématique de la critique féministe libérale contre le multiculturalisme a été présenté par Susan Moller Okin dans son article notoire «Is Multiculturalism Bad for Women? ", Boston Review of Books, 1999. 
La critique de l'approche de l'empowerment (chapitre V) est également saisissante, mais soulève les mêmes questions. Entre le détournement du concept par le pervertissement des discours et des politiques publics, et la corruption théoriquement nécessaire du concept, il importe de faire la part des choses. Car le risque est de reconduire une compréhension tronquée des approches que l'on rejette et de ne pas pouvoir offrir de solutions de rechange véritablement adaptées aux enjeux réels qui nous préoccupent. D’un point de vue strictement théorique, n'aurait-il pas été important d'examiner comment, chez une auteure telle que S. Khader (que l'on soit d'accord ou non avec elle), par exemple, le concept même de l'émancipation est travaillé à l'aune des notions d'autonomie, de préférences adaptatives, de perfectionnisme moral, de fausse conscience, d'aliénation et d'oppression ${ }^{5}$ ? Cela dit, Mestiri a parfaitement raison de dénoncer une forme d'appropriation perverse du discours de l'empowerment au profit d'une conception surplombante culturellement déterminée, c'est-à-dire occidentale, du «féminisme sans frontières" au nom de l'émancipation de toutes les femmes (ainsi en est-il de la thèse West is the Best for Women ${ }^{6}$ ). Mais est-ce le fait d'une récupération politique de ce discours par les ONG et les acteurs principaux du développement international - qui sont généralement issus des pays les plus avantagés et qui reconduiraient donc leurs propres objectifs axiologiques, financiers et programmatiques au nom de l'émancipation -, ou bien le problème résiderait-il plus profondément dans l'appareil théorique luimême?

Or, dans le cadre de ce livre au chapitre VI, il ne paraît pas évident que l'approche du care puisse secourir la notion de l'empowerment sans tomber elle-même dans les travers que Mestiri décrit pourtant si lucidement en invoquant les critiques percutantes de Narayan nous rappelant (parmi d'autres objections redoutables) comment les pouvoirs colonialistes ont tenté de justifier leur domination par le biais d'un discours paternaliste du care $^{7}$. Certains ajouteraient même qu'à comparer les deux approches l'avantage non négligeable de la notion d'empowerment sur celle du care réside dans une compréhension plus positive et dynamique de l'agentivité des femmes, ce que la perspective du care plus attentive aux besoins du bénéficiaire des soins évoque de manière moins intuitive (même si, comme l'auteure le souligne à grands traits, le care conceptualise tout autant sinon davantage le statut de celle/celui qui donne les soins).

5. S. Khader, Adaptive Preferences and Women's Empowerment, Oxford, Oxford University Press, $20 \mathrm{II}$.

6. A. Jagger, «Saving Amina. Global Justice for Women and Intercultural Dialogue ", Ethics and International Affairs, vol, I9, $\mathrm{n}^{\circ} 3,2005$.

7. U. Narayan, "Colonialism and Its Others: Considerations on Rights and Care Discourses », Hypatia, vol. Iо, nº 2, Spring I 995. 
En dernière instance, il aurait été intéressant d'explorer davantage les travaux de F. Robinson ${ }^{8}$, voire même de S. Ruddick (au sujet de «maternal thinking " ${ }^{9}$ ) afin de mieux comprendre comment Mestiri entend surmonter les défis d'un féminisme transculturel à l'aide d'une extrapolation à l'échelle globale de l'approche du care. Dans le sillage des penseures féministes en théorie des relations internationales du côté de la science politique ${ }^{10}$, Robinson propose une critique épistémologique percutante de la conception étatiste du système international ainsi que des grands paradigmes théoriques du réalisme et du libéralisme en vue de promouvoir une éthique internationale ancrée dans les relations d'interdépendance à l'échelle globale. Il va sans dire que la perspective féministe particulière du care recadre le problème de la pauvreté au centre des préoccupations théoriques et pratiques à l'aune des obligations morales interpersonnelles qui précèdent les prérogatives traditionnelles des États. Chez Ruddick, une réflexion critique au sujet de l'approche de la guerre juste conduit à une conception féministe (mais non essentialiste) des vertus du pacifisme dans les domaines de l'activisme politique et de la résolution des conflits. En effet, une des caractéristiques les plus intéressantes des mouvements féministes à travers le monde est le fait qu'en général ils s'inscrivent dans une histoire des luttes sociales qui s'abstiennent (ou ne peuvent) recourir à la violence. On peut être en accord ou non avec ces auteures, mais il aurait été intéressant de savoir ce que Mestiri en pense afin de mieux comprendre en quoi consisteraient les prescriptions normatives d'une approche du care décolonisée à l'échelle internationale.

Mais, de manière générale, les critiques d'une extrapolation globale du care ont du mal à imaginer comment une telle approche pourrait s'appliquer aux «monstres froids» (comme dirait S. Hoffmann) que représentent les États, mus par leurs intérêts égoïstes, en relations internationales. C'est pourquoi d'autres perspectives féministes vont mettre davantage d'importance sur l'analyse des injustices structurelles, genrées et racisées, d'ordre socio-culturel, économique et politique, qu'il importe de mieux comprendre pour mieux défendre et promouvoir les conditions d'existence des femmes à travers le monde, dans toute leur singularité culturelle et particularité contextuelle. C'est à cet égard que les travaux plus récents de la féministe post-colonialiste C. T. Mohanty se révèlent des plus importants pour penser la solidarité féministe "sans frontières» ${ }^{11}$ autour d'une analyse critique du

8. F. Robinson, Globalizing Care. Ethics, Feminist Theory, and International Relations, Boulder, Westview Press, I999.

9. S. Ruddick, Maternal Thinking: Towards a Politics of Peace, Boston, Beacon Press, I989.

10. A. J. Tickner, C. Enloe, H. Charlesworth, etc. (qui ne doivent pas, toutefois, toutes être associées à l'approche du care).

11. C. T. Mohanty, Feminism without Borders. Decolonizing Theory, Practicing Solidarity, Durham \& London, Duke University Press, 2003. 
capitalisme et de ses conséquences structurelles et institutionnelles à l'échelle globale.

Au terme de ces remarques critiques que nous offrons à l'auteure dans le but d'ouvrir une discussion fructueuse, il importe de souligner pourquoi le livre de Soumaya Mestiri est extrêmement important. C'est parce qu'il permet de penser une approche féministe non idéale en philosophie politique, et qui tente réellement de tenir compte des différences profondes et du pluralisme axiologique, du racisme, de l'histoire violente et genrée des rapports d'oppression qui ont déterminé l'état problématique des lieux, qui tente de penser la catégorie de l'universel tout en défiant le paternalisme occidental et l'hégémonie doctrinale. En cela, les travaux de l'auteure contribuent courageusement à la "désobéissance épistémique» qu'elle appelle pour ébranler les biais tenaces et les dogmes conceptuels qui sont véritablement lourds de conséquences politiques que l'on ne peut plus ignorer du haut des tours d'ivoire. 